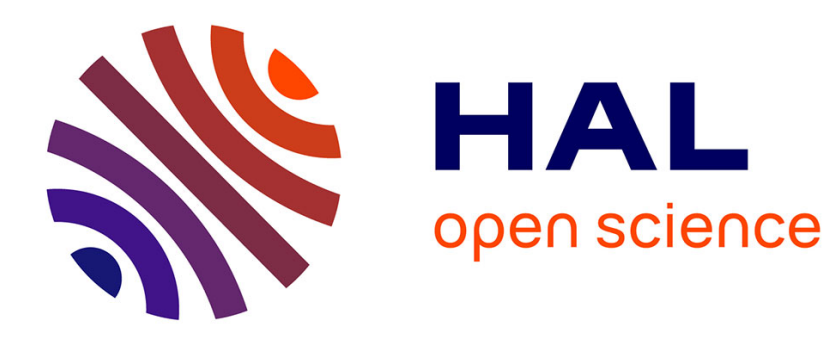

\title{
Correct Proof of Shafer's Belief Conditioning Formulas
}

\author{
Jean Dezert, Albena Tchamova, Deqiang Han
}

\section{To cite this version:}

Jean Dezert, Albena Tchamova, Deqiang Han. Correct Proof of Shafer's Belief Conditioning Formulas. Correct Proof of Shafer's Belief Conditioning Formulas, Nov 2018, XI'AN, China. pp.965-969, 10.1109/cac.2018.8623669 . hal-02335544

\section{HAL Id: hal-02335544 \\ https://hal.science/hal-02335544}

Submitted on 28 Oct 2019

HAL is a multi-disciplinary open access archive for the deposit and dissemination of scientific research documents, whether they are published or not. The documents may come from teaching and research institutions in France or abroad, or from public or private research centers.
L'archive ouverte pluridisciplinaire HAL, est destinée au dépôt et à la diffusion de documents scientifiques de niveau recherche, publiés ou non, émanant des établissements d'enseignement et de recherche français ou étrangers, des laboratoires publics ou privés. 


\section{Correct Proof of Shafer's Belief Conditioning Formulas}

\author{
Jean Dezert \\ The French Aerospace Lab \\ Palaiseau, France. \\ jean.dezert@onera.fr
}

\author{
Albena Tchamova \\ Inst. of I\&C Tech., BAS \\ Sofia, Bulgaria. \\ tchamova@bas.bg
}

\author{
Deqiang Han \\ Inst. of Integrated Automation \\ Xi'an Jiaotong University, China. \\ deqhan@gmail.com
}

\begin{abstract}
In his Mathematical Theory of Evidence published in 1976, Shafer did propose belief and plausibility conditioning formulas based on Dempster's rule of combination. It turns out that the proof given by Shafer for belief conditioning is incorrect and in this paper we present the correct proof of Shafer's belief conditioning formula.
\end{abstract}

Keywords: belief functions, Shafer's conditioning.

\section{INTRODUCTION}

In his Mathematical Theory of Evidence published in 1976 [1], Glenn Shafer did propose belief and plausibility conditioning formulas based on Dempster's rule of combination. It turns out that the proof of Theorem 3.6 given by Shafer in [1] (p. 66) for belief conditioning is incorrect and we will explain why. In this paper we present the correct proof of Shafer's belief conditioning formulas. This paper must not be considered as a support for Shafer's belief conditioning approach because we recommend Fagin-Halpern conditioning approach [2] instead (see our paper [3] for justification). It is only a clarification of correct obtaining of Shafer's conditioning formulas, no less no more.

\section{BASICS OF BELIEF FUNCTIONS}

Based on Dempster's works [4], [5], Shafer did introduce Belief Functions (BF) to model the epistemic uncertainty and to reason under uncertainty [1]. Shafer's theory of evidence is often called Demspter-Shafer Theory (DST) in the literature. We consider a finite discrete frame of discernement (FoD) $\Theta=\left\{\theta_{1}, \ldots, \theta_{n}\right\}$, with $n>1$, and where all exhaustive and exclusive elements of $\Theta$ represent the set of the potential solutions of the problem under concern. The set of all subsets of $\Theta$ is the power-set of $\Theta$ denoted by $2^{\Theta}$. The number of elements (i.e. the cardinality) of $2^{\Theta}$ is $2^{|\Theta|}$. A basic belief assignment (BBA) associated with a given source of evidence is defined as the mapping $m(\cdot): 2^{\Theta} \rightarrow[0,1]$ satisfying the conditions $m(\emptyset)=0$ and $\sum_{A \in 2^{\Theta}} m(A)=1$. The quantity $m(A)$ is the mass of belief of subset $A$ committed by the source of evidence (SoE). A focal element $X$ of a BBA $m(\cdot)$ is an element of $2^{\Theta}$ such that $m(X)>0$. Note that the empty set $\emptyset$ is not a focal element of a BBA because $m(\emptyset)=0$ (closed-world assumption of Shafer's model for the FoD). The set of all focal elements (i.e. the core) of $m(\cdot)$ is denoted $\mathcal{F}_{\Theta}(m) \triangleq\{X \subseteq \Theta \mid m(X)>0\}=\left\{X \in 2^{\Theta} \mid m(X)>0\right\}$, and the set of focal elements of $m(\cdot)$ included in $A \subseteq \Theta$ is denoted $\mathcal{F}_{A}(m) \triangleq\left\{X \in \mathcal{F}_{\Theta}(m) \mid X \cap A=X\right\}$. Belief and plausibility functions are defined by

$$
\begin{aligned}
\operatorname{Bel}(A) & =\sum_{\substack{X \in 2^{\Theta} \\
X \subseteq A}} m(X) \\
& =\sum_{\substack{X \in \mathcal{F}_{\Theta}(m) \\
X \subseteq A}} m(X)=\sum_{X \in \mathcal{F}_{A}(m)} m(X) \\
\operatorname{Pl}(A) & =\sum_{\substack{X \in 2^{\Theta} \\
X \cap A \neq \emptyset}} m(X) \\
& =\sum_{\substack{X \in \mathcal{F}_{\Theta}(m) \\
X \cap A \neq \emptyset}} m(X)=1-\operatorname{Bel}(\bar{A}) .
\end{aligned}
$$

When all elements of $\mathcal{F}_{\Theta}(m)$ are only singletons, $m(\cdot)$ is called a Bayesian BBA [1] and its corresponding $\operatorname{Bel}(\cdot)$ and $P l(\cdot)$ functions are homogeneous to a same (subjective) probability measure $P(\cdot)$. The vacuous BBA representing a totally non informative source of evidence is characterized by the BBA $m(\Theta)=1$. According to Shafer's Theorem 1 (see [1] page 39, with its proof on page 51), the belief functions can be characterized without referencing to a BBA. The quantities $m(\cdot)$ and $\operatorname{Bel}(\cdot)$ are one-to-one, and the BBA $m(\cdot)$ is obtained from $\operatorname{Bel}(\cdot)$ by Möbius inverse formula (see [1], p. 39).

In DST, Shafer [1] did propose to combine $s \geq 2$ distinct sources of evidence represented by BBAs $m_{1}(),. \ldots, m_{s}($. over the same FoD $\Theta$ with Dempster's rule (i.e. the normalized conjunctive rule). Mathematically Dempster's rule of combination of $s \geq 2$ BBAs is defined by $m_{12 \ldots s}^{D S}(\emptyset)=0$, and for any $X \neq \emptyset \in 2^{\Theta}$

$$
\begin{aligned}
m_{12 \ldots s}^{D S}(X) & =\left[m_{1} \oplus \ldots \oplus m_{s}\right](X) \\
& \triangleq m_{12 \ldots s}^{C R}(X) /\left(1-m_{12 \ldots s}^{C R}(\emptyset)\right)
\end{aligned}
$$

where $m_{12 \ldots s}^{C R}(X) \triangleq \sum_{\substack{X_{1}, \ldots, X_{s} \in 2^{\Theta} \\ X_{1} \cap X_{2} \cap \ldots \cap X_{s}=X}} \prod_{i=1}^{s} m_{i}\left(X_{i}\right)$ is the conjunctive rule (CR) of combination. The term $m_{12 \ldots s}^{C R}(\emptyset)$ reflects the amount of dissonance between the sources [6]. Dempster's rule is commutative and associative and preserves

${ }^{1}$ By convention, a sum of non existing terms (if it occurs in formulas depending on the given BBA) is always set to zero. 
the neutrality of vacuous BBA in the fusion process. This rule has been disputed from both theoretical and practical standpoints, see [7]-[13] for discussions. In this paper we do not focus on Dempster's rule, but only on Shafer's belief conditioning formulas based on Demspter's rule.

\section{A. Shafer's conditioning formulas}

In this section we present briefly Shafer's belief conditioning approach as proposed by Shafer in [1]. Suppose that the effect of a new evidence on the frame of discernment $\Theta$ is to establish a particular subset $B \subset \Theta$ with certainty. Then $B e l_{2}$ defined by $\operatorname{Bel}_{2}(A)=1$ if $B \subset A$ and $\operatorname{Bel}_{2}(A)=0$ if $B \not \subset A$ will give a degree of belief one to the proposition corresponding to $B$ and to every proposition implied by it [1], p.66. Shafer established the following important theorem ${ }^{2}$ for conditional belief and plausibility.

Theorem 3.6 [1], p. 67: Suppose $B e l_{2}$ is defined by above two equations, and $B e l_{1}$ is another belief function over $\Theta$. Then $B e l_{1}$ and $B e l_{2}$ are combinable if and only if $\operatorname{Bel}_{1}(\bar{B})<1$. If $B e l_{1}$ and $B e l_{2}$ are combinable, let $\operatorname{Bel}_{1}(\cdot \mid B)$ denote $B e l_{1} \oplus$ $B e l_{2}$, and let $P l_{1}$ and $P l_{1}(\cdot \mid B)$ denote the upper probability functions for $B e l_{1}$ and $B e l_{1} \oplus B e l_{2}$, respectively. Then for all $A \subset \Theta$,

$$
\begin{aligned}
\operatorname{Bel}_{1}(A \mid B) & =\frac{\operatorname{Bel}_{1}(A \cup \bar{B})-B e l_{1}(\bar{B})}{1-B e l_{1}(\bar{B})} \\
P l_{1}(A \mid B) & =\frac{P l_{1}(A \cap B)}{P l_{1}(B)}
\end{aligned}
$$

Shafer's proof of this theorem is in [1] (see pages 71-72), but we reproduce it here for convenience for a better identification of the mistake in this proof.

Shafer's Proof of Theorem 3.6 (as given in [1]): $\operatorname{Bel}_{1}(\bar{B})<$ 1 if and only if $B$ overlaps the core of $B e l_{1}$, and since $B$ is the core of $B e l_{2}$, this is indeed equivalent to $B e l_{1}$ being combinable with $\mathrm{Bel}_{2}$. Denote the basic probability assignments of $B e l_{1}, \mathrm{Bel}_{2}$ and $\mathrm{Bel}_{1} \oplus \mathrm{Bel}_{2}$ by $m_{1}, m_{2}$ and $m$. Since $B$ is the only focal element of $B e l_{2}$, and $m_{2}(B)=1$, Dempster's rule yields

$$
m(A)=\frac{\sum_{\substack{i \\ A_{i} \cap B=A}} m_{1}\left(A_{i}\right)}{1-\sum_{\substack{i \\ A_{i} \cap B=\emptyset}} m_{1}\left(A_{i}\right)}=\frac{\sum_{\substack{C \\ B \cap C=A}} m_{1}(C)}{1-\operatorname{Bel}_{1}(\bar{B})}
$$

and

$$
\begin{aligned}
\operatorname{Bel}_{1}(A \mid B) & =\sum_{D \subset A} m(D)=\frac{\sum_{\substack{D \\
\emptyset \neq D \subset A}} \sum_{C} m_{B} m_{1}(C) D}{1-\operatorname{Bel}_{1}(\bar{B})} \\
& =\frac{\sum_{C} m_{1}(C)}{1-B \cap C \subset A} \\
& =\frac{\sum_{C \subset A \cup \bar{B}} m_{1}(C)}{1-B \not \subset B} \\
= & \frac{B e l_{1}(A \cup \bar{B})-\operatorname{Bel}_{1}(\bar{B})}{1-B e l_{1}(\bar{B})}
\end{aligned}
$$

Hence

$$
\begin{aligned}
P l_{1}(A \mid B) & =1-\operatorname{Bel}_{1}(\bar{A} \mid B) \\
& =\frac{1-\operatorname{Bel}_{1}(\bar{B})-\operatorname{Bel}_{1}(\bar{A} \cup \bar{B})+\operatorname{Bel}_{1}(\bar{B})}{1-\operatorname{Bel}_{1}(\bar{B})} \\
& =\frac{1-\operatorname{Bel}_{1}(\overline{A \cap B})}{1-\operatorname{Bel}_{1}(\bar{B})}=\frac{P l_{1}(A \cap B)}{P l_{1}(B)} .
\end{aligned}
$$

\section{WHY SHAFER'S PROOF IS INCORRECT}

Although Shafer's formulas (4)-(5) are correct $^{3}$, we show why Shafer's proof is incorrect. To obtain the final expression of $\operatorname{Bel}_{1}(A \mid B)$ given by (10), Shafer goes from (8) to (9) in the proof of Theorem 3.6. So, Shafer implicitly assumes that the following equality is valid

$$
\sum_{\substack{C \\ \emptyset \neq B \cap C \subset A}} m_{1}(C)=\sum_{\substack{C \subset A \cup \bar{B} \\ C \not \subset B}} m_{1}(C)
$$

In fact, (14) is wrong as shown in the next simple counterexample. Hence, the Shafer's proof for $\operatorname{Bel}_{1}(A \mid B)$ is incorrect. This mistake casts doubts on the correctness of formulas in Theorem 3.6. However, we show in the next section that formulas given in Theorem 3.6 are in fact correct and we give in this paper their correct proofs. It is quite easy to verify that

\footnotetext{
3 if one accepts Shafer's standpoint for belief conditioning based on Demspster's rule.
}

${ }^{2}$ In his theorem Shafer uses the notation $P^{*}$ for upper probability instea of $P l$ used generally in the literature to denote the plausibility function. 
(9) is not equal to (10) because $^{4}$

$$
\begin{aligned}
& \operatorname{Bel}_{1}(A \cup \bar{B})=\sum_{C \subset A \cup \bar{B}} m_{1}(C) \\
& =\sum_{\substack{C \subset A \cup \bar{B} \\
C \not \subset B}} m_{1}(C)+\sum_{\substack{C \subset A \cup \bar{B} \\
C \subset B}} m_{1}(C) \\
& =\sum_{\substack{C \subset A \cup \bar{B} \\
C \not \subset B}} m_{1}(C)+\sum_{C \subset(A \cup \bar{B}) \cap B} m_{1}(C) \\
& =\sum_{\substack{C \subset A \cup \bar{B} \\
C \not \subset B}} m_{1}(C)+\sum_{C \subset(A \cap B) \cup(\bar{B} \cap B)} m_{1}(C) \\
& =\sum_{\substack{C \subset A \cup \bar{B} \\
C \subset \subset B}} m_{1}(C)+\sum_{C \subset(A \cap B) \cup \emptyset} m_{1}(C) \\
& =\sum_{\substack{C \subset A \cup \bar{B} \\
C \not \subset B}} m_{1}(C)+\sum_{C \subset(A \cap B)} m_{1}(C) \\
& =\sum_{\substack{C \subset A \cup \bar{B} \\
C \not \subset B}} m_{1}(C)+\operatorname{Bel}_{1}(A \cap B)
\end{aligned}
$$

Therefore, the numerators of (9) and (10) are different in general because

$$
\begin{aligned}
\sum_{\substack{C \subset A \cup \bar{B} \\
C \not \subset B}} m_{1}(C) & =\operatorname{Bel}_{1}(A \cup \bar{B})-\operatorname{Bel}_{1}(A \cap B) \\
& \neq \operatorname{Bel}_{1}(A \cup \bar{B})-\operatorname{Bel}_{1}(\bar{B})
\end{aligned}
$$

Remark: One may argue that there is just a small typo error in Shafer's book, and in fact the incorrect expression $\sum_{C \subset A \cup \bar{B}} m_{1}(C)$ in (14), must be replaced by $\sum_{C \subset A \cup \bar{B}} m_{1}(C)$. Even if one admits this possibility of typo error in Shafer's proof, it is not trivial to prove the (modified/corrected) equality

$$
\sum_{\substack{C \\ \emptyset \neq B \cap C \subset A}} m_{1}(C)=\sum_{\substack{C \subset A \cup \bar{B} \\ C \not \subset \bar{B}}} m_{1}(C)
$$

to get the final Shafer's belief conditioning formula. That is why we provide a complete exact and detailed proof of Shafer's belief conditioning formula in section IV.

\section{A simple counter-example of Shafer's proof}

Consider the following FoD $\Theta=\left\{\theta_{1}, \ldots, \theta_{7}\right\}$ satisfying Shafer's model. We consider and denote the focal elements of $m_{1}(\cdot)$ as follows $A \triangleq\left\{\theta_{2}, \theta_{3}, \theta_{4}, \theta_{5}, \theta_{7}\right\}=\theta_{2} \cup \theta_{3} \cup \theta_{4} \cup \theta_{5} \cup \theta_{7}$, $B \triangleq\left\{\theta_{1}, \theta_{2}, \theta_{3}, \theta_{4}\right\}=\theta_{1} \cup \theta_{2} \cup \theta_{3} \cup \theta_{4}, C_{1} \triangleq\left\{\theta_{3}, \theta_{5}, \theta_{6}\right\}=$ $\theta_{3} \cup \theta_{5} \cup \theta_{6}, C_{2} \triangleq\left\{\theta_{4}, \theta_{7}\right\}=\theta_{4} \cup \theta_{7}, C_{3} \triangleq \theta_{2}$, and the BBA $m_{1}($.$) defined on the FoD \Theta$ given by $m_{1}(A)=0.1$, $m_{1}(B)=0.1, m_{1}\left(C_{1}\right)=0.2, m_{1}\left(C_{2}\right)=0.3$ and $m_{1}\left(C_{3}\right)=$ 0.3 . We consider the subset $B=\theta_{1} \cup \theta_{2} \cup \theta_{3} \cup \theta_{4}$ being the conditioning term, characterized by the BBA $m_{2}(B)=1$,

\footnotetext{
${ }^{4}$ The denominators of (9) and (10) being equal, we just need to verify if the numerators of (9) and (10) are equal, or not.
}

hence $\operatorname{Bel}_{2}(B)=1$. Note that $\bar{B}=\Theta \backslash B=\left\{\theta_{5}, \theta_{6}, \theta_{7}\right\}$ and $\operatorname{Bel}_{1}(\bar{B})=0$ because there is no focal elements of $m_{1}(\cdot)$ included in $\bar{B}=\theta_{5} \cup \theta_{6} \cup \theta_{7}$.

- Let us calculate at first the sum $S_{1} \triangleq \sum_{\emptyset \neq B \cap C \subset A} m_{1}(C)$ involved in (8). All focal elements $C$ of $m_{1}(\cdot)$ such that $\emptyset \neq$ $B \cap C \subset A$ are the focal elements $A, C_{1}, C_{2}$ and $C_{3}$ because $B \cap A=\theta_{2} \cup \theta_{3} \cup \theta_{4} \neq \emptyset$ and $\theta_{2} \cup \theta_{3} \cup \theta_{4} \subset A, B \cap$ $C_{1}=\theta_{3} \neq \emptyset$ and $\theta_{3} \subset A, B \cap C_{2}=\theta_{4} \neq \emptyset$ and $\theta_{4} \subset A$, $B \cap C_{3}=\theta_{2} \neq \emptyset$ and $\theta_{2} \subset A$. The focal element $C=B$ of $m_{1}(\cdot)$ is not involved in the sum $S_{1}$ because if $C=B$, then $B \cap C=B \cap B=B \not \subset A$. Therefore, one gets

$$
\begin{aligned}
S_{1} & =m_{1}(A)+m_{1}\left(C_{1}\right)+m_{1}\left(C_{2}\right)+m_{1}\left(C_{3}\right) \\
& =0.1+0.2+0.3+0.3=0.9
\end{aligned}
$$

Hence, based on (8) which is the correct expression obtained from (7), one gets the correct value of Shafer's belief conditioning

$$
\operatorname{Bel}_{1}(A \mid B)=S_{1} /\left(1-\operatorname{Bel}_{1}(\bar{B})\right)=0.9 /(1-0)=0.9
$$

- Let us calculate the sum $S_{2} \triangleq \sum_{C \subset A \cup \bar{B}} m_{1}(C)$ involved in (9). First note that $A \cup \bar{B}=\theta_{2} \cup \theta_{3} \cup \theta_{4} \cup \theta_{5} \cup \theta_{6} \cup \theta_{7}$ and the focal elements $C$ of $m_{1}(\cdot)$ such that $C \subset(A \cup \bar{B})$ and $C \not \subset B$ are the three focal elements $A, C_{1}$ and $C_{2}$ because $A \subset A \cup \bar{B}$ and $A \not \subset B, C_{1}=\theta_{3} \cup \theta_{5} \cup \theta_{6} \subset A \cup \bar{B}$ and $C_{1} \not \subset B, C_{2}=\theta_{4} \cup \theta_{7} \subset A \cup \bar{B}$ and $C_{2} \not \subset B$. The focal element $B=\theta_{1} \cup \theta_{2} \cup \theta_{3} \cup \theta_{4}$ of $m_{1}(\cdot)$ is not included in $A \cup \bar{B}=\theta_{2} \cup \theta_{3} \cup \theta_{4} \cup \theta_{5} \cup \theta_{6} \cup \theta_{7}$ because, in this example, $B$ is not included in $A$, and of course because $B \cap \bar{B}=\emptyset$. The focal element $C_{3}=\theta_{2}$ of $m_{1}(\cdot)$ is included in $A \cup \bar{B}=$ $\theta_{2} \cup \theta_{3} \cup \theta_{4} \cup \theta_{5} \cup \theta_{6} \cup \theta_{7}$ but $C_{3}=\theta_{2}$ is also included in $B=\theta_{1} \cup \theta_{2} \cup \theta_{3} \cup \theta_{4}$, so that the condition $C_{3} \not \subset B$ is not satisfied. Based on these remarks, one gets for $S_{2}$

$$
S_{2}=m_{1}(A)+m_{1}\left(C_{1}\right)+m_{1}\left(C_{2}\right)=0.1+0.2+0.3=0.6
$$

We can verify that the value of $S_{2}$ corresponds to the value obtained with the correct formula (??), because $\operatorname{Bel}_{1}(A \cup \bar{B})=$ $m_{1}(A)+m_{1}\left(C_{1}\right)+m_{1}\left(C_{2}\right)+m_{1}\left(C_{3}\right)=0.9$ and $\operatorname{Bel}_{1}(A \cap$ $B)=m_{1}\left(C_{3}\right)=0.3$ so that $S_{2}=\operatorname{Bel}_{1}(A \cup \bar{B})-\operatorname{Bel}_{1}(A \cap$ $B)=0.9-0.3=0.6$. Hence, based on (9), one would get an incorrect value of Shafer's belief conditioning

$$
\operatorname{Bel}_{1}(A \mid B)=S_{2} /\left(1-\operatorname{Bel}_{1}(\bar{B})\right)=0.6 /(1-0)=0.6
$$

Clearly, this counter-example shows that $S_{1} \neq S_{2}$ and proves that the equality (14) is incorrect. This simple counter examples illustrates that the proof of Theorem 3.6 given by Shafer is incorrect. 


\section{CORRECT PROOF OF FORMULAS OF THEOREM 3.6}

Starting from Dempster's rule we have $m(\emptyset)=0$ and for all $A \neq \emptyset \in 2^{\Theta}$,

$$
m(A)=\left[m_{1} \oplus m_{2}\right](A)=\frac{\sum_{\substack{X_{1}, X_{2} \in 2^{\Theta} \\ X_{1} \cap X_{2}=A}} m_{1}\left(X_{1}\right) m_{2}\left(X_{2}\right)}{1-\sum_{\substack{X_{1}, X_{2} \in 2^{\Theta} \\ X_{1} \cap X_{2}=\emptyset}} m_{1}\left(X_{1}\right) m_{2}\left(X_{2}\right)}
$$

Because in conditioning by $B \neq \emptyset, m_{2}(\cdot)$ is defined by $m_{2}\left(X_{2}\right)=1$ if $X_{2}=B$ and $m_{2}\left(X_{2}\right)=0$ otherwise, the previous expression reduces for $A \neq \emptyset$ to

$$
m(A)=\frac{\sum_{\substack{X_{1} \in 2^{\Theta} \\ \emptyset \neq X_{1} \cap B=A}} m_{1}\left(X_{1}\right)}{1-\sum_{\substack{X_{1} \in 2^{\Theta} \\ X_{1} \cap B=\emptyset}} m_{1}\left(X_{1}\right)}=\frac{\sum_{\substack{X_{1} \in 2^{\Theta} \\ \emptyset \neq X_{1} \cap B=A}} m_{1}\left(X_{1}\right)}{1-\operatorname{Bel}_{1}(\bar{B})}
$$

because $\operatorname{Bel}_{1}(\bar{B})=\sum_{\substack{X_{1} \in 2^{\Theta} \\ X_{1} \subseteq \bar{B}}} m_{1}\left(X_{1}\right)=\sum_{\substack{X_{1} \in 2^{\Theta} \\ X_{1} \cap B=\emptyset}} m_{1}\left(X_{1}\right)$.

Using the definition of the belief function, $\operatorname{Bel}_{1}(A \mid B)$ for $B \neq$ $\emptyset$ is given by

$$
\begin{aligned}
\operatorname{Bel}_{1}(A \mid B) & =\sum_{\substack{Y \in 2^{\Theta} \\
Y \subseteq A}} m(Y) \\
& =\sum_{\substack{Y \in 2^{\Theta} \\
Y \subseteq A}} \frac{\sum_{\substack{X_{1} \in 2^{\Theta} \\
\emptyset \neq X_{1} \cap B=Y}} m_{1}\left(X_{1}\right)}{1-B e l_{1}(\bar{B})} \\
& =\frac{\sum_{Y \in 2^{\Theta}} \sum_{\substack{X_{1} \in 2^{\Theta} \\
\emptyset \neq X_{1} \cap B=Y}} m_{1}\left(X_{1}\right)}{\sum_{\substack{X_{1} \in 2^{\ominus} \\
\emptyset \neq X_{1} \cap B \subseteq A}} m_{1}\left(X_{1}\right)} \\
& =\frac{B e l_{1}(\bar{B})}{1-B \bar{B})}
\end{aligned}
$$

Note that equation (18) is the same as Shafer's equation (8) using slight modified notations ${ }^{5}$ for better presentation in the sequel.

Because $m_{1}(\cdot)$ is a normalized BBA, one has for all $B \in 2^{\Theta}$

$$
\sum_{\substack{X_{1} \in 2^{\Theta} \\ X_{1} \cap B=\emptyset}} m_{1}\left(X_{1}\right)+\sum_{\substack{X_{1} \in 2^{\ominus} \\ X_{1} \cap B \neq \emptyset}} m_{1}\left(X_{1}\right)=1
$$

Also, for any $A \in 2^{\Theta}$ and in partitioning $2^{\Theta}$ in the subsets $\left\{Y \in 2^{\Theta} \mid Y \subseteq A\right\}$ and $\left\{Y \in 2^{\Theta} \mid Y \nsubseteq A\right\}$, the following

\footnotetext{
${ }^{5}$ We have also replaced symbol $\subset$ by $\subseteq$ for clarity.
}

equality also always holds

$$
\begin{aligned}
& \sum_{\substack{Y \in 2^{\Theta} \\
Y \subseteq A}}\left[\sum_{\substack{X_{1} \in 2^{\Theta} \\
X_{1} \cap B \cap Y=\emptyset}} m_{1}\left(X_{1}\right)+\sum_{\substack{X_{1} \in 2^{\Theta} \\
X_{1} \cap B \cap Y \neq \emptyset}} m_{1}\left(X_{1}\right)\right] \\
& +\sum_{\substack{Y \in 2^{\Theta} \\
Y \nsubseteq A}}\left[\sum_{\substack{X_{1} \in 2^{\Theta} \\
X_{1} \cap B \cap Y=\emptyset}} m_{1}\left(X_{1}\right)+\sum_{\substack{X_{1} \in 2^{\Theta} \\
X_{1} \cap B \cap Y \neq \emptyset}} m_{1}\left(X_{1}\right)\right]=1
\end{aligned}
$$

This equality can be rewritten equivalently as

$$
\begin{aligned}
& \sum_{\substack{X_{1} \in 2^{\ominus} \\
\left(X_{1} \cap B=\emptyset\right) \subseteq A}} m_{1}\left(X_{1}\right)+\sum_{\substack{X_{1} \in 2^{\ominus} \\
\left(X_{1} \cap B \neq \emptyset\right) \subseteq A}} m_{1}\left(X_{1}\right) \\
& +\sum_{\substack{X_{1} \in 2^{\Theta} \\
\left(X_{1} \cap B=\emptyset\right) \unrhd A}} m_{1}\left(X_{1}\right)+\sum_{\substack{X_{1} \in 2^{\Theta} \\
\left(X_{1} \cap B \neq \emptyset\right) \subseteq A}} m_{1}\left(X_{1}\right)=1
\end{aligned}
$$

The second term of the left hand side of (21) corresponds to the numerator of $\operatorname{Bel}_{1}(A \mid B)$ given in (18). We can express it as

$$
\begin{gathered}
\sum_{\substack{X_{1} \in 2^{\Theta} \\
\left(X_{1} \cap B \neq \emptyset\right) \subseteq A}} m_{1}\left(X_{1}\right)=1-\sum_{\substack{X_{1} \in 2^{\Theta} \\
\left(X_{1} \cap B=\emptyset\right) \subseteq A}} m_{1}\left(X_{1}\right) \\
-\sum_{\substack{X_{1} \in 2^{\Theta} \\
\left(X_{1} \cap B=\emptyset\right) \unrhd A}} m_{1}\left(X_{1}\right)-\sum_{\substack{X_{1} \in 2^{\Theta} \\
\left(X_{1} \cap B \neq \emptyset\right) \unrhd A}} m_{1}\left(X_{1}\right)
\end{gathered}
$$

Because

$$
\sum_{\substack{X_{1} \in 2^{\Theta} \\\left(X_{1} \cap B=\emptyset\right) \subseteq A}} m_{1}\left(X_{1}\right)+\sum_{\substack{X_{1} \in 2^{\Theta} \\\left(X_{1} \cap B=\emptyset\right) \subseteq A}} m_{1}\left(X_{1}\right)=\sum_{\substack{X_{1} \in 2^{\Theta} \\ X_{1} \cap B=\emptyset}} m_{1}\left(X_{1}\right)
$$

one gets

$$
\begin{aligned}
\sum_{\substack{X_{1} \in 2^{\Theta} \\
\left(X_{1} \cap B \neq \emptyset\right) \subseteq A}} m_{1}\left(X_{1}\right) & =1-\sum_{\substack{X_{1} \in 2^{\ominus} \\
X_{1} \cap B=\emptyset}} m_{1}\left(X_{1}\right) \\
& -\sum_{\substack{X_{1} \in 2^{\ominus} \\
\left(X_{1} \cap B \neq \emptyset\right) \notin A}} m_{1}\left(X_{1}\right) \\
& =1-\operatorname{Bel}_{1}(\bar{B})-P l_{1}(\bar{A} \cap B)
\end{aligned}
$$

The last previous equality comes from the fact that

$$
\begin{gathered}
\operatorname{Bel}_{1}(\bar{B})=\sum_{\substack{X_{1} \in 2^{\Theta} \\
X_{1} \subseteq \bar{B}}} m_{1}\left(X_{1}\right)=\sum_{\substack{X_{1} \in 2^{\Theta} \\
X_{1} \cap B=\emptyset}} m_{1}\left(X_{1}\right) \\
P l_{1}(\bar{A} \cap B)=\sum_{\substack{X_{1} \in 2^{\Theta} \\
X_{1} \cap B \cap \bar{A} \neq \emptyset}} m_{1}\left(X_{1}\right)=\sum_{\substack{X_{1} \in 2^{\ominus} \\
\left(X_{1} \cap B \neq \emptyset\right) \notin A}} m_{1}\left(X_{1}\right)
\end{gathered}
$$

Therefore, the numerator of $\operatorname{Bel}_{1}(A \mid B)$ given in (18) equals $1-P l_{1}(\bar{A} \cap B)-B e l_{1}(\bar{B})$. Because $P l_{1}(\bar{A} \cap B)=1-$ $\operatorname{Bel}_{1}(\overline{\bar{A} \cap B})=1-\operatorname{Bel}_{1}(A \cup \bar{B})$, one finally gets for the numerator of $\operatorname{Bel}_{1}(A \mid B)$

$$
\sum_{\substack{X_{1} \in 2^{\Theta} \\\left(X_{1} \cap B \neq \emptyset\right) \subset A}} m_{1}\left(X_{1}\right)=\operatorname{Bel}_{1}(A \cup \bar{B})-\operatorname{Bel}_{1}(\bar{B})
$$


and the final expression of $\operatorname{Bel}_{1}(A \mid B)$ is given by

$$
\operatorname{Bel}_{1}(A \mid B)=\left(\operatorname{Bel}_{1}(A \cup \bar{B})-\operatorname{Bel}_{1}(\bar{B})\right) /\left(1-\operatorname{Bel}_{1}(\bar{B})\right)
$$

This expression coincides with the final expression (10) given by Shafer in his flawed proof. The derivation of $\mathrm{Pl}_{1}(A \mid B)$ given in Shafer's proof is correct since we have proved that the expression of $\operatorname{Bel}_{1}(A \mid B)$ is correct.

\section{CONCLUSion}

In this paper we have shown why the proof of belief conditioning formulas given by Shafer is wrong and we have illustrated this incorrectness with a simple counter-example. After the identification of the mistake in Shafer's proof, we have provided the correct proof of final expressions of Shafer's belief conditioning formulas. For readers interested in belief conditioning, we provide a solid justification against the belief conditioning method proposed by Shafer in our companion paper [3]. Our criticism of Shafer's conditioning approach is based on the Total Belief Theorem and Generalized Bayes' Theorem.

\section{REFERENCES}

[1] Shafer, G.: A Mathematical Theory of Evidence, Princeton University Press, 1976.

[2] Fagin, R., Halpern J.Y.: A new approach to updating beliefs, UAI Proc., 1991.

[3] Dezert, J., Tchamova, A., Han, D.: Total Belief Theorem and Generalized Bayes' Theorem, Fusion 2018 Proc., Cambridge, UK, July 2018.

[4] Dempster, A.: Upper and lower probabilities induced by a multivalued mapping, Ann. of Math. Stat., (38):325-339, 1967.

[5] Dempster, A.: A generalization of Bayesian inference, J. Roy. Stat. Soc., B30, 1968.

[6] D. Dubois, H. Prade, A set-theoretic view of belief functions, Int. J. General Systems, Vol. 12, pp. 193-226, 1976.

[7] Zadeh, L.: A Mathematical Theory of Evidence (book review), AI Mag. (5), 1984.

[8] Lemmer, J.: Confidence factors, empiricism and the Dempster-Shafer theory of evidence, Proc. of 1st UAI Conf., pp. 16-176, 1985.

[9] Black, P.K.: Is Shafer general Bayes?, 3rd UAI Workshop, USA, 1987.

[10] Wang, P.: A defect in Dempster-Shafer theory, UAI Conf. Proc., 1994.

[11] Dezert, J., Wang, P., Tchamova, A.: On the validity of Dempster-Shafer theory, Fusion 2012 Proc., Singapore, July 9-12, 2012.

[12] Dezert, J., Tchamova, A., Han, D., Tacnet, J.-M.: Why Dempster's fusion rule is not a generalization of Bayes fusion rule, Fusion Proc., Turkey, July 2013.

[13] Dezert, J., Tchamova, A.: On the validity of Dempster's fusion rule and its interpretation as a generalization of Bayesian fusion rule, Int. J. of Intell. Syst., (29):223-252, 2014. 